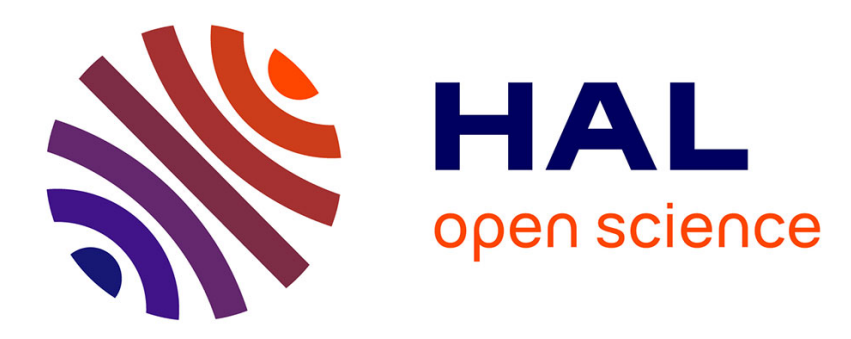

\title{
Cycles intersecting edge-cuts of prescribed sizes
}

Tomáš Kaiser, Riste Škrekovski

\section{To cite this version:}

Tomáš Kaiser, Riste Škrekovski. Cycles intersecting edge-cuts of prescribed sizes. 2005 European Conference on Combinatorics, Graph Theory and Applications (EuroComb '05), 2005, Berlin, Germany. pp.303-308, 10.46298/dmtcs.3465 . hal-01184454

\section{HAL Id: hal-01184454 \\ https://hal.inria.fr/hal-01184454}

Submitted on 14 Aug 2015

HAL is a multi-disciplinary open access archive for the deposit and dissemination of scientific research documents, whether they are published or not. The documents may come from teaching and research institutions in France or abroad, or from public or private research centers.
L'archive ouverte pluridisciplinaire HAL, est destinée au dépôt et à la diffusion de documents scientifiques de niveau recherche, publiés ou non, émanant des établissements d'enseignement et de recherche français ou étrangers, des laboratoires publics ou privés. 


\title{
Cycles intersecting edge-cuts of prescribed sizes
}

\author{
Tomáš Kaiser ${ }^{1,3 \dagger}$ and Riste Škrekovski ${ }^{2,3 \ddagger}$ \\ ${ }^{1}$ Department of Mathematics, University of West Bohemia, Univerzitni 8, 30614 Plzeř, Czech Republic. \\ E-mail: kaisertekma.zcu.cz. \\ ${ }^{2}$ Department of Mathematics, University of Ljubljana, Jadranska 19, 1111 Ljubljana, Slovenia. \\ E-mail: skreko@fmf.uni-1j.si. \\ ${ }^{3}$ Institute for Theoretical Computer Science (ITI), Charles University, Praha, Czech Republic.
}

We prove that every cubic bridgeless graph $G$ contains a 2-factor which intersects all (minimal) edge-cuts of size 3 or 4 . This generalizes an earlier result of the authors, namely that such a 2 -factor exists provided that $G$ is planar. As a further extension, we show that every graph contains a cycle (a union of edge-disjoint circuits) that intersects all edge-cuts of size 3 or 4 . Motivated by this result, we introduce the concept of a coverable set of integers and discuss a number of questions, some of which are related to classical problems of graph theory such as Tutte's 4-flow conjecture or the Dominating circuit conjecture.

Keywords: graph, cycle, edge-cut, covering cycle, coverable set

\section{Introduction}

We study the existence of cycles intersecting all edge-cuts of prescribed sizes in a graph. Throughout this paper, a cycle in a graph $G$ is a union of edge-disjoint circuits and an edge-cut (in short, a cut) is an inclusionwise minimal set of edges whose removal increases the number of components of $G$. Our graphs are undirected and contain no loops, but they may contain parallel edges.

Our starting point is the main result of [6]:

Theorem 1.1 For any planar graph $G$, there exists a (not necessarily proper) 2-coloring of $V(G)$ such that there is no monochromatic circuit of length 3 or 4.

In an equivalent dual form, Theorem 1.1 states that every bridgeless planar cubic graph has a 2-factor intersecting all cuts of size 3 or 4 . (A graph is bridgeless if it is connected and has no bridges.) In the present paper, we extend the latter result to all bridgeless cubic graphs. Furthermore, we remove the regularity assumption, proving the following:

\footnotetext{
${ }^{\dagger}$ Supported by project 1M0021620808 and Research Plan MSM 4977751301 of the Czech Ministry of Education

${ }^{\ddagger}$ Supported in part by the Ministry of Science and Technology of Slovenia, Research Program P1-0297, and by project 1M0021620808 of the Czech Ministry of Education.
} 
Theorem 1.2 Every graph $G$ has a cycle intersecting all cuts of size 3 or 4.

Motivated by this, we introduce the following concept. Let $\mathbb{N}$ be the set of positive integers and $A \subseteq \mathbb{N}$. We say that a cycle $C$ in a graph $G$ is $A$-covering if it intersects all cuts $T$ with $|T| \in A$. If $\mathcal{Q}$ is a class of graphs, then $A$ is coverable in $\mathcal{Q}$ if every graph from $\mathcal{Q}$ contains an $A$-covering cycle. A set that is coverable in the class of all graphs is just said to be coverable.

Thus, an equivalent version of Theorem 1.2 is that the set $\{3,4\}$ is coverable. Which other sets are coverable? $\mathbb{N}$ itself is not; clearly, a graph has an $\mathbb{N}$-covering cycle if and only if it has a spanning Eulerian subgraph (spanning closed trail), which is not the case, for instance, for the graph $K_{2,3}$ (or for any graph with a bridge). In fact, $K_{2,3}$ shows that even the set $\{2\}$ is not coverable.

For a less trivial example of a non-coverable set, consider $A=\{3,5\}$ and the Petersen graph $P_{10}$. For any vertex $v$ of $P_{10}$, the edges incident with $v$ constitute a cut as $P_{10}$ is 3 -edge-connected. Since $3 \in A$, any $A$-covering cycle is a 2 -factor. Every 2 -factor $F$ of $P_{10}$ is formed by two circuits of length 5 . The complement of $F$ is a cut of size 5 that is not intersected by $F$. It follows that $P_{10}$ has no $A$-covering cycle.

On the other hand, it may well be that the presence of $P_{10}$ in a graph $G$ (as a minor) is the only obstruction to the existence of a $\{3,5\}$-covering cycle in $G$. Recall that a graph $H$ is a minor of a graph $G$ if $H$ can be obtained from $G$ by a sequence of edge contractions and edge deletions. The graph $G$ is Petersen-minor-free (or $P_{10}$-free) if $P_{10}$ is not a minor of $G$. Petersen-minor-free graphs are the subject of the famous 4-flow conjecture of Tutte [12]. Since we will not need to go into the details of integer flows (which can be found in [13]), let us state Tutte's conjecture in a form that does not refer to 4-flows:

Conjecture 1.3 Any $P_{10}$-free bridgeless graph $G$ contains cycles $C_{1}, C_{2}$ such that $E(G)=E\left(C_{1}\right) \cup$ $E\left(C_{2}\right)$.

Observe that if $E(G)$ is the union of the edge sets of cycles $C_{1}$ and $C_{2}$, then $C_{1}$ is $(2 \mathbb{N}+1)$-covering, where $2 \mathbb{N}+1=\{3,5,7, \ldots\}$. Indeed, any odd cut not intersected by $C_{1}$ cannot be contained in $C_{2}$, for the intersection of a cycle and a cut has even size. Conversely, it is not difficult to prove that if $G$ has a $(2 \mathbb{N}+1)$-covering cycle, then $E(G)$ is the union of edge sets of two cycles. Thus, Conjecture 1.3 can equivalently be stated in terms of coverability:

Conjecture 1.4 The set $2 \mathbb{N}+1=\{3,5,7, \ldots\}$ is coverable in the class of $P_{10}$-free graphs.

Observe that Conjecture 1.4 is not restricted to bridgeless graphs. The reason is that any set $A \subseteq \mathbb{N}$ with $1 \notin A$ is coverable in the class of bridgeless graphs if and only if it is coverable in the class of all graphs.

Conjecture 1.3 is well known to be true for planar graphs. Indeed, this special case is equivalent to the Four Color Theorem (see, e.g., [13]). It follows that $2 \mathbb{N}+1$ is coverable in the class of planar graphs.

To conclude this section, we point out a relation to another long-standing conjecture. A subgraph $H$ of a graph $G$ is dominating if each edge of $G$ is incident with a vertex of $H$. The Dominating circuit conjecture has several equivalent forms [2, 7, 10]; we state the one due to Fleischner and Jackson [4] (see Section 2 for a definition of cyclically $k$-connected graphs):

Conjecture 1.5 Every cyclically 4-edge-connected cubic graph has a dominating circuit.

By Tutte's theorem [11], Conjecture 1.5 is true for planar graphs. Note that if $G$ is a cyclically 4-edgeconnected cubic graph, then a circuit is dominating in $G$ if and only if it is an $(\mathbb{N}+3)$-covering cycle, where $\mathbb{N}+3=\{4,5,6, \ldots\}$. Thus, the following is a generalization of the Dominating circuit conjecture: 
Conjecture 1.6 The set $\mathbb{N}+3$ is coverable.

A result of Thomassen [9, Theorem 4.1] implies that $\mathbb{N}+3$ is coverable in the class of planar graphs. Further questions related to coverable sets are asked in Section 4.

\section{Notation and definitions}

Let us review the necessary definitions. As mentioned above, the graphs we consider are loopless multigraphs. The vertex set and the edge set of a graph $G$ are denoted by $V(G)$ and $E(G)$, respectively. If $E=E(G)$, we write $E(v)$ for the set of edges incident with the vertex $v$.

Recall that a cut in a connected graph $G$ is a subset $C \subseteq E(G)$ such that $G-C$ is disconnected and $C$ is minimal with this property. Note that $G-C$ has two components, say, $G_{1}$ and $G_{2}$. If any of the graphs $G_{i}$ consists of a single vertex, then $C$ is a trivial cut; otherwise $C$ is called non-trivial. Similarly, if any of the graphs $G_{i}$ is a tree, then $C$ is an acyclic cut; otherwise $C$ is cyclic. We refer to edge cuts of size $k$ as $k$-cuts.

A graph $G$ is cyclically $k$-edge-connected if $|E(G)|>k$ and $G$ has no cyclic cut of size at most $k-1$. A cycle $H$ is spanning in $G$ if each vertex of $G$ is incident with an edge of $H$.

\section{$3\{3,4\}$-covering cycles}

By a well-known theorem of Petersen, every bridgeless cubic graph $G$ has a 2 -factor. In this section, we prove a result which implies that in fact, $G$ has a 2 -factor which is a $\{3,4\}$-covering cycle. To this end, we make use of the following extension of the Petersen theorem, due to Schönberger [8]:

Theorem 3.1 Let $G$ be a cubic bridgeless multigraph and e, $f \in E(G)$. Then $G$ has a 2-factor containing both e and $f$.

Let $v$ be a vertex of degree 4 in a graph $G=(V, E)$. Let $Y \subset E(v)$ be a set of size 2 and let $X \subseteq E(v)$ be an even set (a set of even size). Note that if $|X| \neq 2$, then $X=E(v)$. We say that $X$ crosses $Y$ if $X \cap Y \neq \emptyset$ and $Y \neq X$. Thus, $E(v)$ crosses each of its subsets of size 2 .

Example 3.2 If $E(v)=\{a, b, c, d\}$ and $Y=\{a, b\}$, then the even sets which cross $Y$ are $\{a, c\},\{a, d\}$, $\{b, c\},\{b, d\}$ and $\{a, b, c, d\}$.

Let $w \in V(G)$. We say that a subgraph $H \subseteq G$ extends a set $X \subseteq E(w)$ if $E(H) \cap E(w)=X$.

The following theorem deals with $\{3,4\}$-covering cycles in graphs with maximum degree at most 4 , the focus being on cubic graphs where any such cycle is a 2 -factor.

Theorem 3.3 Let $G$ be a 2-connected graph and let $v$ be a vertex of $G$. Suppose that $v$ is of degree at most 4 and all the other vertices of $G$ are of degree at most 3.

(a) If $v$ is of degree at most 3 , then each set $Y \subset E(v)$ with $|Y|=2$ can be extended to a $\{3,4\}$ covering cycle of $G$.

(b) If $v$ is of degree 4, then there exists a set $X \subset E(v)$ of size 2 such that every even set $Y \subseteq E(v)$ which crosses $X$ can be extended to a $\{3,4\}$-covering cycle of $G$.

For instance, in the situation of Example 3.2, part (b) of the theorem claims that each of the sets $\{a, c\}$, $\{a, d\},\{b, c\},\{b, d\}$, and $\{a, b, c, d\}$ can be extended to a $\{3,4\}$-covering cycle of $G$. 
Corollary 3.4 Every bridgeless graph with maximum degree at most 3 has a $\{3,4\}$-covering cycle.

Theorem 3.3 implies the following strengthening of Theorem 3.1:

Corollary 3.5 Every cubic bridgeless graph has a 2-factor which intersects all cuts of size 3 and 4. Moreover, any two incident edges can be extended to such a 2-factor.

Theorem 1.2 can be easily deduced from Corollary 3.5 using Fleischner's Splitting Lemma [3].

\section{Remarks}

By Theorem 1.2, both the sets $\{3\}$ and $\{4\}$ are coverable. On the other hand, $\{1\}$ and $\{2\}$ are not. How about the other single-element sets?

Question 4.1 Is it true that for all $k \geq 3,\{k\}$ is coverable?

We are unable to say anything for $k \geq 5$, except that Conjecture 1.6 clearly implies an affirmative answer to this question. On the other hand, since (as we noted in Section 1) the conjecture is true for planar graphs, any set $\{k\}$ is coverable in the class of planar graphs (which will be denoted by $\mathcal{P}$ throughout this section).

Having determined which sets of size 1 are coverable in $\mathcal{P}$, we may attempt the same for sets of size 2. Let $A=\{a, b\}$ be a pair of positive integers with $a<b$. If $a \leq 2$ then $A$ is not coverable in $\mathcal{P}$, and if $a \geq 4$, then the planar case of Conjecture 1.6 implies that $A$ is coverable in $\mathcal{P}$. Thus, we may assume that $a=3$. Since the set $2 \mathbb{N}+1=\{3,5,7, \ldots\}$ is coverable in $\mathcal{P}$ (by the Four Color Theorem), we may assume that $b$ is even and $b \geq 6$.

Question 4.2 Let $k \geq 3$. Is $\{3,2 k\}$ coverable in $\mathcal{P}$ ?

In fact, this is an equivalent form of a question posed by Broersma et al. [1] in connection with Theorem 1.1: For which $k \geq 3$ can one 2-color the vertices of every planar graph in such a way that there is no monochromatic circuit of length 3 or $2 k$ ? To our knowledge, the question is open. It may even be that $\{3,2 k\}(k \geq 3)$ is coverable in the class of all graphs.

One might speculate that even the set consisting of 3 and all the numbers $2 k(k \geq 2)$ is coverable. It can be shown that this is not the case:

Proposition 4.3 The set $A=\{3,4,6,8,10, \ldots\}$ is not coverable.

Our argument proving Proposition 4.3 uses a non-hamiltonian 3-connected cubic bipartite graph. It cannot be applied in the class $\mathcal{P}$, for there is no known example of a planar graph with these properties. Indeed, a well-known conjecture of D. Barnette [5, Section 2.12] states that there is no such graph. Thus, we conclude our paper with the following question:

Question 4.4 Is $\{3,4,6,8,10, \ldots\}$ coverable in $\mathcal{P}$ ?

\section{References}

[1] H. Broersma, F. V. Fomin, J. Kratochvíl and G. J. Woeginger, Planar graph coloring avoiding monochromatic subgraphs: trees and paths make things difficult, Algorithmica, to appear. 
[2] H. Fleischner, Cycle decompositions, 2-coverings, removable cycles, and the four-color-disease, in: J. A. Bondy and U. S. R. Murty (eds.), Progress in Graph Theory, Academic Press, 1984, pp. 233-246.

[3] H. Fleischner, Eine gemeinsame Basis für die Theorie der eulerschen Graphen und den Satz von Petersen, Monatsh. Math. 81 (1976), 267-278.

[4] H. Fleischner and B. Jackson, A note concerning some conjectures on cyclically 4-edge-connected 3-regular graphs, in: L. D. Andersen et al. (eds.), Graph Theory in Memory of G. A. Dirac, Ann. Discrete Math., vol. 41, North-Holland, Amsterdam, 1989, pp. 171-178.

[5] T. R. Jensen and B. Toft, Graph Coloring Problems, J. Wiley \& Sons, New York, 1995.

[6] T. Kaiser and R. Škrekovski, Planar graph colorings without short monochromatic cycles, J. Graph Theory 46 (2004), 25-38.

[7] M. M. Matthews and D. P. Sumner, Hamiltonian results in $K_{1,3}$-free graphs, J. Graph Theory 8 (1984), 139-146.

[8] T. Schönberger, Ein Beweis des Petersenschen Graphensatzes, Acta Litt. Sci. Szeged 7 (1934), 5157.

[9] C. Thomassen, Decomposing a planar graph into degenerate graphs, J. Combin. Theory Ser. B 65 (1995), 305-314.

[10] C. Thomassen, Reflections on graph theory, J. Graph Theory 10 (1986), 309-324.

[11] W. T. Tutte, A theorem on planar graphs, Trans. Amer. Math. Soc. 82 (1956), 99-116.

[12] W. T. Tutte, On the algebraic theory of graph colourings, J. Combin. Theory 1 (1966), 15-50.

[13] C.-Q. Zhang, Integer Flows and Cycle Covers of Graphs, Dekker, New York, 1997. 
